\title{
IL-6, through p-STAT3 rather than p-STAT1, activates hepatocarcinogenesis and affects survival of hepatocellular carcinoma patients: a cohort study
}

Jung-Ta Kao ${ }^{1,2,3}$, Chun-Lung Feng ${ }^{3}$, Cheng-Ju Yu ${ }^{3}$, Shu-Mei Tsai ${ }^{4}$, Ping-Ning Hsu ${ }^{5}$, Yao-Li Chen ${ }^{6,7 \dagger}$ and Yi-Ying $\mathrm{Wu}^{2,8^{*}+}$

\begin{abstract}
Background: Biologic activities of functional mediators activate downstream transducers regulating inflammation and carcinogenesis. Correlation among mediators (IL-6, IL-27, TNF-a, and VEGF) with STAT proteins at diverse clinical-pathologic stages of hepatocellular carcinoma (HCC) remains limited.

Methods: Serum mediators assayed from 147 untreated HCC cases (HCC-total group) included 70 HBV-infected (HCC-HBV group), 64 HCV-infected (HCC-HCV group), and 13 without HBV-/HCV-infection (HCC-NBNC group). Another 156 non-HCC individuals comprised 54 healthy individuals (HG) and 102 chronic hepatitis patients (CH-total group) as control group. To correlate with serum mediators, 86-paired liver tissues (CH: 52 and HCC: 34 cases) served for p-STATs proteins immunostain.

Results: Although four mediators (IL-6, IL-27, TNF-a, and VEGF) significantly over-expressed, IL-6 presented the strongest correlation in HCC-total versus $\mathrm{CH}$-total or HG groups ( $\mathrm{HCC}$-total versus $\mathrm{CH}$-total: $\mathrm{P}<0.001$; HCC-total versus HG: $P<0.001$ ). Over-expressed IL-6 concentration linked with poor liver function (Albumin: $r=-0.383, P<$ 0.001; Bilirubin: $r=0.280, P=0.001$; INR: $r=0.299, P<0.001$; AST: 0.212, $P=0.016$ ), tumor progression (TNM system: $r=0.370 ; P<0.001$ ), clinical condition severity (BCLC system: $r=0.471 ; P<0.001$; terminal- versus early-stage HCC, $P=0.001$; advanced- versus early-stage HCC, $P=0.007$; terminal- versus intermediate- stage HCC $P=0.003$; advanced- versus intermediate-stage HCC $P=0.019)$, and 6-month mortality $(P=0.024)$. Likewise, serum IL-6 $(r=0.501$, $P=0.003)$ as compared to IL-27 $(r=0.052, P=0.770)$, TNF- $a(r=0.019, P=0.917)$, and VEGF $(r=0.096, P=0.595)$ expression reflected positive correlation with activation of tissues $\mathrm{p}$-STAT3 rather than p-STAT1.
\end{abstract}

Conclusions: Serum IL-6, through p-STAT3 rather than p-STAT1 signal pathway, affected hepatic function, tumor progression, and determine HCC patient survival.

Keywords: IL-6, p-STAT3, Chronic hepatitis, Hepatocellular carcinoma

\footnotetext{
* Correspondence: yyw@mail.cmu.edu.tw

${ }^{\dagger}$ Equal contributors

${ }^{2}$ Graduate Institute of Clinical Medical Science, China Medical University,

Taichung, Taiwan

${ }^{8}$ Department of Medical Laboratory Science and Biotechnology, China

Medical University, No. 91, Hsueh-Shih Rd., Taichung 404, Taiwan

Full list of author information is available at the end of the article
} 


\section{Background}

Worldwide, 711,000 new hepatocellular carcinoma (HCC) cases are diagnosed per annum, with 679,000 eventually dying [1]. Hepatitis B (HBV) and C virus (HCV) infections both contribute as leading causes [2,3]. Diagnostic and therapeutic modalities have emerged in clinical scenarios; to date, these prove inadequate due to obstacles of vascular invasion or extra-hepatic metastases [4-6]. Biochemical candidates have thus been identified by cell line or animal studies that contribute to early development and distant spread of cancer cells, but are rarely available in clinical applications [7-11]. The possibility could arise from the complicated interactions between tumor and host microenvironment in the real world. Therefore, through clinical evidence, to find the effective biomarkers and further clarify interactions with their downstream signaling targets should help greatly when evaluating actual roles in clinical settings and finally devising effective therapeutic strategies to solve this global problem.

Among well-recognized mediators, wide-ranging biological activities by IL-6, IL-27, TNF- $\alpha$, and VEGF have been implicated in regulating inflammation and/or carcinogenesis [12-16]. In hepatic study, multifunctional cytokine IL-6 can stimulate hepatocyte proliferation and regeneration as well as growth modulation and tumor differentiation. High IL-6 levels might reflect more active hepatic necro-inflammation and associate with severity of disease [17-19]. Interleukin-27 (IL-27), a heterodimeric cytokine belonging to the IL-12 family, not only act on hepatocytes against viral activity but also curb tumor proliferation $[20,21]$. Tumor necrosis factor-alpha (TNF- $\alpha)$, regarded as a pro-inflammatory cytokine, is actively involved in regulation of portal hypertension and carcinogenesis [22-24]. In contrast to multifunctional activities of IL- 6 and IL-27 and TNF- $\alpha$ in different stages of liver disease, vascular endothelial growth factor (VEGF), an essential regulator during angiogenesis rather than inflammatory process [15,25], triggers blood vessel growth for nutrition of cancer cells and affects survival in advanced HCC cases [26-28]. However, biologic function of these mediators is mediated by signaling pathways. Among them, signal transducers and activators of transcription (STATs) have been observed as essential components linking cytokine signals to transcriptional events that lead to cell proliferation, protection from apoptosis, tumorigenesis, and increased metastatic potential in various cells, including cancer [12,16,29-32]. Yet the relationship and biologic effect of functional mediators with STATs proteins is limited mostly to study of cell lines, animal models, or non-HCC patients, and is poorly understood in clinical HCC patients.

To elucidate clinical roles and relationships of IL-6, IL-27, TNF- $\alpha$, and VEGF with STATs proteins at different clinical-pathological stages of HCC, we conducted this cohort study. Findings on biological mechanisms of these molecules and their interrelations with cancer might increase our understanding to create new therapeutic modalities for managing liver tumors.

\section{Methods \\ Patients}

With informed consent, 303 patients with well-characterized clinical conditions for serum mediators assay, including 147 naïve HCC patients (HCC-total group), 102 chronic hepatitis patients (CH-total group) and 54 healthy persons (HG) were enrolled in China Medical University Hospital at Taichung, Taiwan. As per clinical serological diagnoses, HCC-total patients were subgrouped as: (1) 70 with positive HBsAg for longer than 6 months (HCC-HBV), (2) 64 with positive anti-HCV $\mathrm{Ab}$ for more than 6 months (HCC-HCV), and (3) 13 with negative $\mathrm{HBsAg}$ and anti-HCV markers (HCCNBNC group). Hepatitis patients without $\mathrm{HCC}$ but with positive HBsAg or anti-HCV marker for longer than 6 months were enrolled as the chronic hepatitis $(\mathrm{CH})$ group: $28 \mathrm{HBV}$ - and $74 \mathrm{HCV}$-infected. Those, without $\mathrm{HCC}$ and negative $\mathrm{HBsAg} /$ anti-HCV marker, were enrolled as a healthy group (HG). Another 86-paired liver tissues, including $34 \mathrm{HCC}(12 \mathrm{HBV}, 17 \mathrm{HCV}$, and 5 NBNC cases) plus $52 \mathrm{CH}$ (18 HBV and $34 \mathrm{HCV}$ cases) served for immunostain of STAT1 (p-STAT1) and STAT3 phosphorylation (p-STAT3).

The HCC was defined as: (1) histopathology proven by liver biopsy, or (2) image such as abdomen computerized tomography showing HCC diagnosis. Classification of HCC severity accorded with TNM and the Barcelona Clinic Liver Cancer (BCLC) staging system, as did treatment of all HCC cases. Additionally, according to median HCC survival [11] as well as rate of BCLC severity and mortality in our study, we selected 6-month mortality as cut-off point and evaluated the correlation with four mediators. Patients with (1) co-infection or superinfection (HBV or HCV); (2) prior antiviral agents like interferon or nucleoside analogues, immunomodulatory or anti-tumour agent; (3) autoimmune hepatitis or druginduced liver disease; or (4) acute inflammation within two weeks, such as gout arthritis, were excluded. Procedures conformed to ethical standards of the responsible Committee on Human Experimentation (institutional and national) and with the 1975 Helsinki Declaration, as revised in 2008. The Institutional Review Board of China Medical University Hospital also approved this study.

\section{Serological virus markers and liver biochemical assays methodology}

Serum HBV markers, anti-HCV antibodies, HBV DNA, and HCV RNA levels were assessed by commercial enzyme immunoassay (AxSYM, Abbott, North Chicago, IL; 
Abbott HCV EIA 2.0; Abbott Laboratories; Cobas Amplicor HCV Monitor 2.0; Roche Diagnostics, Branchburg, NJ). Albumin, AFP, ALT, AST, bilirubin, coagulation, and creatinine were tested by autoanalyzer (TBA-30FR, Toshiba; Tokyo, Japan).

\section{Estimation of serum mediators and tissue immunohistochemistry}

Venous blood samples were obtained from a peripheral vein of all enrolled cases and immediately centrifuged, plasma stored at $-80{ }^{\circ} \mathrm{C}$. Quantification of IL-6, IL-27, TNF- $\alpha$ and VEGF by specific ELISA used commercially available kits within two weeks (IL-6, IL-27, and TNF- $\alpha$ used by eBioscience, San Diego, CA; VEGF used by Antigenix American, Huntington Station, NY). Results were expressed in picograms per milliliter $(\mathrm{pg} / \mathrm{ml})$, liver tissues fixed in $10 \%$ formalin and embedded in paraffin. Blocks were sectioned at $4 \mu \mathrm{m}$ for each tissue and three pieces of each specimen stained, including one without and two with phosphorylation according to standard protocol (Cell Signaling Technology, Inc. 3 Trask Lane, Danvers, MA). The p-STAT1 and p-STAT3 immunostaining was assessed quantitatively by counting the total number of positively stained cytoplasma and nuclei of hepatocytes per 10 high-power fields ( $\times 400$ magnifications) microscopically from each specimen. Positive immunostain was considered when $\geqq 10 \%$ nuclei or cytoplasma of hepatocytes were stained [33]. The immunoreactivity expression was categorized as Level I (mean $<10 \%$ nuclei or cytoplasma of hepatocytes stained, II (mean $\geqq 10 \%$ to $<25 \%$ nuclei or cytoplasma of hepatocytes stained, or III (mean $\geqq 25 \%$ nuclei or cytoplasma of hepatocytes stained).

\section{Statistical analysis}

Baseline data were expressed as mean \pm standard deviation (Table) and mean \pm standard error deviation (figures and mediators), each group of experiments repeated at least twice to confirm data. Continuous variables were assessed by Student $t$-test and Pearson correlation, data analyzed by SPSS version 17.0 for Microsoft Windows (SPSS, Chicago, IL). Two-sided $P$-value $<0.05$ indicated statistical significance.

\section{Results}

\section{Patients' demographic and clinical characteristics}

Table 1 shows baseline characteristics of 147 HCC, 102 hepatitis cases, and 54 healthy persons. HCC-total patients were older than those in $\mathrm{CH}$-total and $\mathrm{HG}$ groups $(65.36 \pm 11.68$ versus $50.20 \pm 14.53$ versus $42.87 \pm 13.35$ years respectively), which was compatible with distribution of liver diseases.

Compared to healthy group (HG), four mediators besides VEGF presented significantly in $\mathrm{CH}$ group

In $\mathrm{HBV}$ - or HCV-infected liver disease, IL-6, IL-27, and TNF- $\alpha$ presented significant expression in $\mathrm{CH}$-total group than HG $(3.79 \pm 1.40$ versus $0.46 \pm 0.10 \mathrm{pg} / \mathrm{ml}$ in IL-6, $\mathrm{P}=0.02 ; 164.19 \pm 33.43$ versus $9.83 \pm 3.26 \mathrm{pg} / \mathrm{ml}$ in IL-27, $\mathrm{P}<0.001 ; 75.62 \pm 28.14$ versus $1.76 \pm 1.49 \mathrm{pg} / \mathrm{ml}$ in TNF- $\alpha, \mathrm{P}=0.011$ respectively) (Figure 1 ).

Among four mediators, over-expression of IL- 6 presented the strongest correlation with HCC-total and separate HCC groups than healthy $(\mathrm{HG})$ or $\mathrm{CH}$-total group

While hepatocarcinogenesis, four mediators presented predominant expression in HCC-total group as compared to HG $(19.70 \pm 3.28$ versus $0.46 \pm 0.10 \mathrm{pg} / \mathrm{ml}$ in IL-6, $\mathrm{P}<0.001 ; 298.46 \pm 69.23$ versus $9.83 \pm 3.26 \mathrm{pg} / \mathrm{ml}$ in IL-27, $\mathrm{P}<0.001 ; 65.40 \pm 23.82$ versus $1.76 \pm 1.49 \mathrm{pg} /$ $\mathrm{ml}$ in TNF- $\alpha, \mathrm{P}=0.009 ; 14.12 \pm 2.91$ versus $0.40 \pm$ $0.07 \mathrm{pg} / \mathrm{ml}$ in VEGF, $\mathrm{P}<0.001$ respectively) but TNF- $\alpha$ failed in $\mathrm{CH}$ group $(19.70 \pm 3.28$ versus $3.79 \pm 1.40 \mathrm{pg} /$ $\mathrm{ml}$ in IL-6, $\mathrm{P}<0.001 ; 298.46 \pm 69.23$ versus $164.19 \pm$ $33.43 \mathrm{pg} / \mathrm{ml}$ in IL-27, $\mathrm{P}=0.040 ; 65.40 \pm 23.82$ versus $75.62 \pm 28.14 \mathrm{pg} / \mathrm{ml}$ in TNF- $\alpha, \mathrm{P}=0.792 ; 14.12 \pm 2.91$ versus $1.79 \pm 1.12 \mathrm{pg} / \mathrm{ml}$ in VEGF, $\mathrm{P}=0.001$ respectively). Furthermore, IL- 6 presented the strongest expression in separate HCC groups: HCC-HBV group versus $H G(15.57 \pm 3.85$ versus $0.46 \pm 0.10 \mathrm{pg} / \mathrm{ml}$ in IL-6, $\mathrm{P}<0.001 ; 240.39 \pm 95.39$ versus $9.83 \pm 3.26 \mathrm{pg} / \mathrm{ml}$ in IL-27, $\mathrm{P}=0.018 ; 24.74 \pm 9.36$ versus $1.76 \pm 1.49 \mathrm{pg} /$ $\mathrm{ml}$ in TNF- $\alpha, \mathrm{P}=0.018 ; 19.02 \pm 5.39$ versus $0.40 \pm$ $0.07 \mathrm{pg} / \mathrm{ml}$ in VEGF, $\mathrm{P}=0.001$ respectively) and $\mathrm{CH}-\mathrm{HBV}$ group $(15.57 \pm 3.85$ versus $6.55 \pm 4.31 \mathrm{pg} / \mathrm{ml}$ in IL-6, $\mathrm{P}=0.174 ; 240.39 \pm 95.39$ versus $139.61 \pm$ $40.51 \mathrm{pg} / \mathrm{ml}$ in IL-27, $\mathrm{P}=0.504 ; 24.74 \pm 9.36$ versus $4.57 \pm 1.49 \mathrm{pg} / \mathrm{ml}$ in TNF- $\alpha, P=0.124 ; 19.02 \pm 5.39$ versus $2.15 \pm 1.58 \mathrm{pg} / \mathrm{ml}$ in VEGF, $\mathrm{P}=0.046$ respectively); HCC-HCV group versus HG (23.96 \pm 5.98 versus $0.46 \pm 0.10 \mathrm{pg} / \mathrm{ml}$ in IL-6, $\mathrm{P}<0.001 ; 325.98 \pm 98.96$ versus $9.83 \pm 3.26 \mathrm{pg} / \mathrm{ml}$ in IL-27, $\mathrm{P}=0.002 ; 119.77 \pm$ 53.11 versus $1.76 \pm 1.49 \mathrm{pg} / \mathrm{ml}$ in $\mathrm{TNF}-\alpha, \mathrm{P}=0.030$ $9.53 \pm 2.77$ versus $0.40 \pm 0.07 \mathrm{pg} / \mathrm{ml}$ in VEGF, $\mathrm{P}=0.002$ respectively) and $\mathrm{CH}-\mathrm{HCV}$ group $(23.96 \pm 5.98$ versus $2.78 \pm 1.06 \mathrm{pg} / \mathrm{ml}$ in IL-6, $\mathrm{P}=0.001 ; 325.98 \pm 98.96$ versus $175.53 \pm 44.12 \mathrm{pg} / \mathrm{ml}$ in IL-27, $\mathrm{P}=0.169 ; 119.77 \pm$ 53.11 versus $76.35 \pm 28.52 \mathrm{pg} / \mathrm{ml}$ in TNF- $\alpha, \mathrm{P}=0.43$; $9.53 \pm 2.77$ versus $1.65 \pm 0.78 \mathrm{pg} / \mathrm{ml}$ in VEGF, $\mathrm{P}=0.066$ respectively); and $\mathrm{HCC}-\mathrm{NBNC}$ group versus $\mathrm{HG}$ $(21.14 \pm 9.16$ versus $0.46 \pm 0.10 \mathrm{pg} / \mathrm{ml}$ in IL-6, $\mathrm{P}<$ $0.001 ; 482.75 \pm 353.68$ versus $9.83 \pm 3.26 \mathrm{pg} / \mathrm{ml}$ in IL$27, \mathrm{P}=0.003 ; 16.05 \pm 7.41$ versus $1.76 \pm 1.49 \mathrm{pg} / \mathrm{ml}$ in TNF $-\alpha, P=0.083 ; 10.09 \pm 5.96$ versus $0.40 \pm 0.07 \mathrm{pg} / \mathrm{ml}$ in VEGF, $\mathrm{P}=0.001$ respectively) (Figure 1 ).

Among four mediators, over-expression of IL-6 correlated with deterioration of liver and tumor condition according to the BCLC staging system

Of four mediators, higher IL-6 level not only presented the strongest correlation with clinical factors in liver 
Table 1 Baseline characteristics of healthy and chronic hepatitis $(\mathrm{CH})$ and hepatocellular carcinoma $(\mathrm{HCC})$ patients $(\mathrm{N}=303)$

\begin{tabular}{|c|c|c|c|c|c|}
\hline \multirow[t]{2}{*}{ Demographics } & \multicolumn{2}{|l|}{ Non-HCC group $(n=156)$} & \multicolumn{3}{|l|}{ HCC group $(n=147)$} \\
\hline & Healthy group $(n=54)$ & $\mathrm{CH}$ group $(n=102)$ & HCC-NBNC $(n=13)$ & HCC-HBV $(n=70)$ & HCC-HCV $(n=64)$ \\
\hline Age (yrs) (range) & $42.87 \pm 13.35(19-68)$ & $50.20 \pm 14.53(19-78)$ & $70.38 \pm 8.85(57-85)$ & $60.00 \pm 11.91(30-86)$ & $69.36 \pm 9.71(47-86)$ \\
\hline Sex (Male) (\%) & $33(61.10 \%)$ & $61(59.8 \%)$ & $9(69.20 \%)$ & $56(80.00 \%)$ & $37(57.80 \%)$ \\
\hline Cirrhosis & 0 & $13(12.7 \%)$ & $9(69.20 \%)$ & $47(67.10 \%)$ & $44(68.80 \%)$ \\
\hline $\begin{array}{l}\text { Varices/Variceal } \\
\text { bleeding (\%) }\end{array}$ & $0 / 0$ & $3 / 0$ & $6 / 1$ & $33 / 11$ & $27 / 13$ \\
\hline $\begin{array}{l}\text { BCLC system (Early/ } \\
\text { Intermediated/Advanced/ } \\
\text { Terminal Stage) }\end{array}$ & $-/-/-1-$ & $-/-/-1-$ & $0 / 3 / 7 / 3$ & $14 / 10 / 24 / 22$ & 13/19/17/15 \\
\hline \multicolumn{6}{|l|}{ Biochemical values } \\
\hline Albumin (g/dL) & $4.44 \pm 0.37(3.5-5.0)$ & $4.22 \pm 0.51(2.30-5.10)$ & $3.32 \pm 0.56(2.40-4.50)$ & $3.33 \pm 0.73(2.00-4.90)$ & $3.26 \pm 0.63(2.00-4.80)$ \\
\hline Bilirubin (mg/dL) & $0.80 \pm 0.23(0.25-1.30)$ & $1.04 \pm 0.50(0.42-4.11)$ & $3.42 \pm 5.58(0.44-19.84)$ & $2.77 \pm 3.42(0.38-16.47)$ & $1.97 \pm 2.52(0.47-14.67)$ \\
\hline Creatinine (mg/dL) & $0.83 \pm 0.20(0.49-1.51)$ & $0.86 \pm 0.31(0.43-2.62)$ & $1.47 \pm 0.86(0.61-3.43)$ & $1.10 \pm 0.99(0.42-6.95)$ & $1.50 \pm 1.60(0.47-10.66)$ \\
\hline AST (IU/L) & $21.86 \pm 6.28(14-51)$ & $66.58 \pm 66.16(12-463)$ & $121.10 \pm 162.74(29.00-578.00)$ & $166.88 \pm 428.01(23.00-3410.00)$ & $106.51 \pm 106.86(25.00-488.00)$ \\
\hline ALT (IU/L) & $20.93 \pm 7.16(10-40)$ & $86.07 \pm 137.34(13-1330)$ & $48.38 \pm 28.49(5.00-103.00)$ & $73.11 \pm 105.62(16.00-633.00)$ & $72.14 \pm 60.97(10.00-366.00)$ \\
\hline INR & $0.96 \pm 0.06(0.86-1.18)$ & $1.02 \pm 0.08(0.87-1.42)$ & $1.25 \pm 0.41(0.85-2.21)$ & $1.27 \pm 0.46(0.89-4.50)$ & $1.15 \pm 0.22(0.86-2.20)$ \\
\hline Platelet $\left(10^{3} / \mathrm{uL}\right)$ & $245.46 \pm 57.22(138-351)$ & $179.01 \pm 58.24(44-378)$ & $163.62 \pm 66.73(22.00-265.00)$ & $172.93 \pm 91.03(21.00-451.00)$ & $124.50 \pm 59.86(18.00-323.0)$ \\
\hline $\operatorname{AFP}(\mathrm{ng} / \mathrm{mL})$ & $2.78 \pm 1.45(1.0-8.02)$ & $21.92 \pm 79.29(0.99-611.11)$ & $15421.25 \pm 23754.14(2.55-54001.0)$ & $11119.34 \pm 20318.09(2.05-54001.00)$ & $5817.04 \pm 15840.43(1.33-54001.0)$ \\
\hline \multicolumn{6}{|l|}{ Virologic values } \\
\hline HBeAg (+) (\%) & 0 & 9 (9/28 = 32.14\%) & 0 & 7 (10\%) & 0 \\
\hline
\end{tabular}




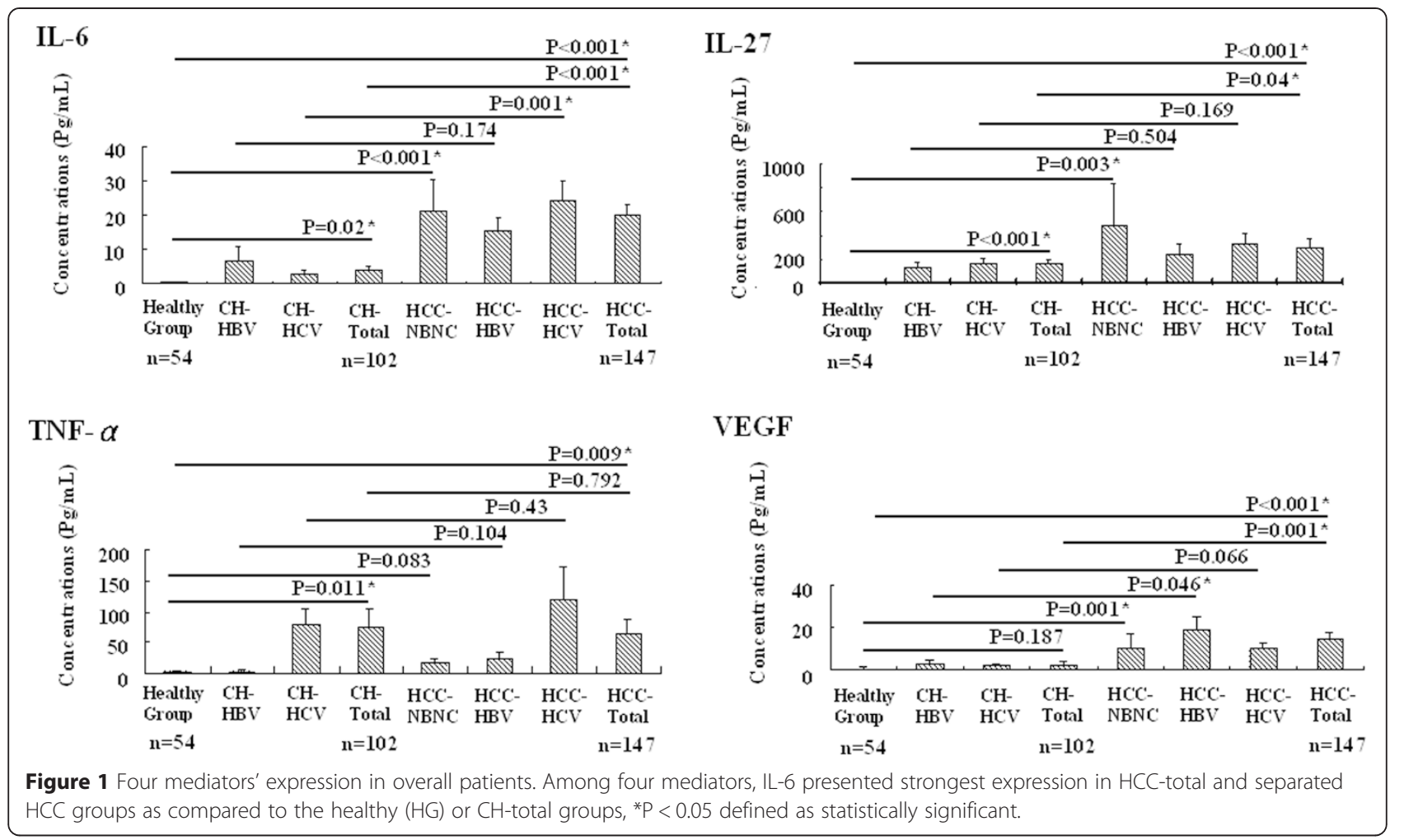

function-e.g., Albumin $(\mathrm{r}=-0.383 ; \mathrm{P}<0.001)$, AST $(\mathrm{r}=$ 0.212; $\mathrm{P}=0.016)$, Bilirubin $(\mathrm{r}=0.280 ; \mathrm{P}=0.001)$, INR $(\mathrm{r}=$ 0.299 ; $\mathrm{P}<0.001$ ) (Table 2) - but also proved significant in deteriorating patient condition, as per the BCLC scoring system (terminal- versus early-stage HCC: $31.28 \pm$ 7.14 versus $4.87 \pm 1.66 \mathrm{pg} / \mathrm{ml}, \mathrm{P}=0.001$; terminal- versus intermediate-stage HCC: $31.28 \pm 7.14$ versus $7.61 \pm 2.84 \mathrm{pg} /$ $\mathrm{ml}, \mathrm{P}=0.003$; advanced- versus early-stage HCC: $27.70 \pm$ 7.83 versus $4.87 \pm 1.66 \mathrm{pg} / \mathrm{ml}, \mathrm{P}=0.007$; advanced- versus intermediate-stage HCC $27.70 \pm 7.83$ versus $7.61 \pm 2.84 \mathrm{pg} /$ $\mathrm{ml}, \mathrm{P}=0.019)$. Conversely, IL-27, TNF- $\alpha$, and VEGF could not present significance in each stage (Figure 2).

Table 2 Correlations between IL-6, IL-27, TNF- $a$, and VEGF with biochemical data in HCC patients

\begin{tabular}{|c|c|c|c|c|c|c|c|c|}
\hline & \multicolumn{2}{|l|}{ IL-6 } & \multicolumn{2}{|l|}{ IL-27 } & \multicolumn{2}{|l|}{ TNF- $a$} & \multicolumn{2}{|l|}{ VEGF } \\
\hline & $\gamma$ & $P$ & $\gamma$ & $P$ & $\gamma$ & $P$ & $\Gamma$ & $P$ \\
\hline Age (years) & 0.137 & 0.106 & -0.016 & 0.855 & -0.013 & 0.882 & -0.078 & 0.363 \\
\hline Sex (F/M) & 0.057 & 0.504 & -0.052 & 0.538 & -0.215 & $0.011 *$ & 0.023 & 0.788 \\
\hline Varices $(-/+)$ & 0.155 & 0.067 & 0.024 & 0.775 & 0.058 & 0.495 & 0.152 & 0.075 \\
\hline Variceal bleeding $(-/+)$ & 0.135 & 0.112 & 0.042 & 0.625 & 0.215 & $0.011 *$ & 0.154 & 0.071 \\
\hline Albumin (g/dL) & -0.383 & $<0.001 *$ & -0.098 & 0.253 & -0.031 & 0.717 & -0.175 & $0.041 *$ \\
\hline Bilirubin (mg/dL) & 0.280 & $0.001^{*}$ & 0.046 & 0.590 & 0.161 & 0.060 & -0.112 & 0.175 \\
\hline Creatinine (mg/dL) & 0.181 & $0.032^{*}$ & -0.037 & 0.663 & 0.022 & 0.792 & -0.074 & 0.391 \\
\hline AST (IU/L) & 0.212 & $0.016^{*}$ & 0.112 & 0.208 & 0.092 & 0.300 & 0.020 & 0.819 \\
\hline ALT (IU/L) & -0.087 & 0.305 & 0.083 & 0.329 & 0.087 & 0.305 & -0.084 & 0.325 \\
\hline INR & 0.299 & $<0.001 *$ & -0.064 & 0.460 & -0.095 & 0.269 & 0.160 & 0.063 \\
\hline Platelet $\left(10^{3} / \mathrm{uL}\right)$ & 0.036 & 0.671 & 0.043 & 0.619 & 0.016 & 0.857 & 0.002 & 0.985 \\
\hline AFP (ng/mL) & 0.261 & $0.002^{*}$ & 0.002 & 0.977 & 0.120 & 0.157 & 0.123 & 0.152 \\
\hline TNM Staging & 0.370 & $<0.001^{*}$ & -0.109 & 0.200 & -0.052 & 0.539 & 0.059 & 0.491 \\
\hline BCLC Staging & 0.471 & $<0.001 *$ & -0.028 & 0.739 & 0.035 & 0.682 & 0.158 & 0.065 \\
\hline
\end{tabular}

$(\mathrm{N}=147) * \mathrm{p}<0.05$ defined as significant 

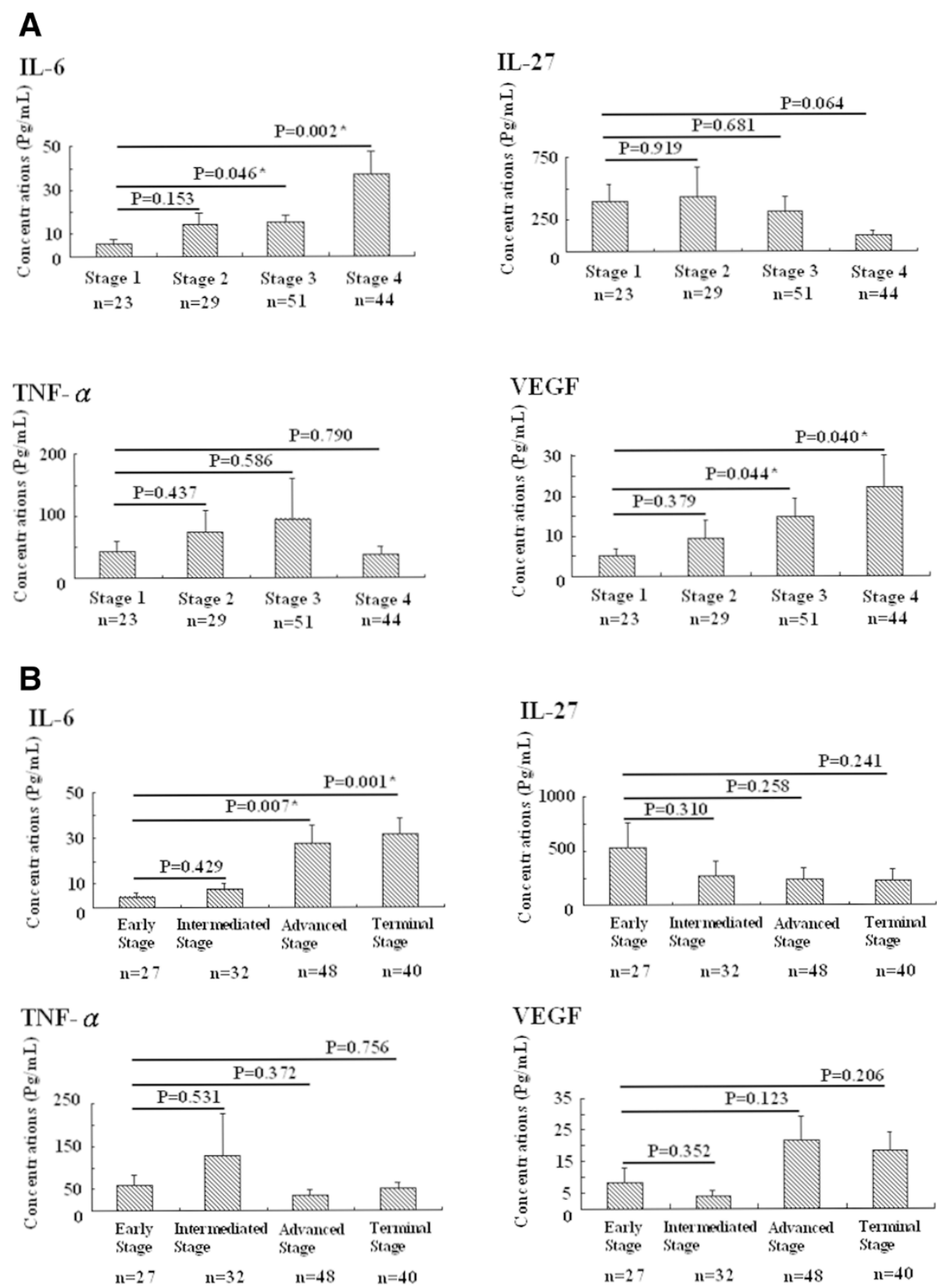

Figure 2 Four mediators' expression in tumor severity. Among the four mediators, IL-6 and VEGF showed significant expressions in patients with stages 3 or 4 HCC according to the classifications of TNM staging system (Figure 2A), but only IL-6 showed a significant difference in patients with advanced or terminal-stage HCC according to classification of the BCLC staging system (Figure $2 \mathrm{~B}$ ), ${ }^{*} \mathrm{P}<0.05$ defined as significant.

\section{Among four mediators, IL-6 over-expression predicted obviously 6-month mortality}

Compatible with the above, over-expression of IL- 6 correlated with mortality ( $<6$-month versus $\geq 6$-month survival: $29.66 \pm 5.86$ versus $12.23 \pm 3.51 \mathrm{pg} / \mathrm{ml}, \mathrm{P}=0.012$, respectively) while portending 6-month mortality in HCC-total cases (Table 3). By contrast, elevated IL-27, TNF- $\alpha$, or VEGF presented no significant correlation with 6-month mortality (Figure 3).
Liver preservation (Child-Pugh Classification) rather than presence or absence of cirrhosis presented high correlation with IL-6 over-expression

There was no link between four mediators with presence or not of cirrhosis $(19.80 \pm 4.03$ versus $19.50 \pm 5.70 \mathrm{pg} /$ $\mathrm{ml}$ in IL-6, $\mathrm{P}=0.966 ; 299.30 \pm 79.70$ versus $296.67 \pm$ $135.87 \mathrm{pg} / \mathrm{ml}, \mathrm{P}=0.986$ in $\mathrm{IL}-27 ; 74.13 \pm 34.09$ versus $46.98 \pm 17.98 \mathrm{pg} / \mathrm{ml}$ in TNF- $\alpha, \mathrm{P}=0.596 ; 12.92 \pm 2.80$ versus $16.60 \pm 6.82 \mathrm{pg} / \mathrm{ml}$ in VEGF, $\mathrm{P}=0.555$ respectively). 
Table 3 Univariate cox regression model of baseline characteristics and 6-month mortality in hepatocellular carcinoma (HCC) patients

\begin{tabular}{lcccc}
\hline Variable & Parameter estimate & SE of estimate & Risk ratio $(95 \% \mathrm{Cl})$ & \\
\hline Demographics & & & & \\
Age (yrs) & 0.011 & 0.016 & $1.011(0.981-1.043)$ & 0.465 \\
Gender (F/M) & 0.532 & 0.392 & $1.702(0.790-3.668)$ & 0.174 \\
Varices (-/+) & 1.490 & 0.381 & $4.438(2.101-9.373)$ & $<0.001^{*}$ \\
Variceal rupture (-/+) & 1.496 & 0.493 & $4.463(1.697-11.739)$ & $0.002^{*}$ \\
IL-6 (pg/mL) & 0.015 & 0.007 & $1.015(1.002-1.028)$ & $0.024^{*}$ \\
IL-27 (pg/mL) & 0.000 & 0.000 & $1.000(0.999-1.000)$ & 0.204 \\
TNF-a (pg/mL) & -0.001 & 0.001 & $0.999(0.996-1.002)$ & 0.401 \\
VEGF (pg/mL) & 0.034 & 0.013 & $1.034(1.009-1.060)$ & $0.007^{*}$ \\
AST (IU/L) & 0.008 & 0.003 & $1.008(1.003-1.013)$ & $0.003^{*}$ \\
ALT (IU/L) & 0.002 & 0.002 & $1.002(0.998-1.007)$ & 0.337 \\
Albumin (g/dL) & -2.413 & 0.444 & $0.090(0.038-0.214)$ & $<0.001^{*}$ \\
Bilirubin (mg/dL) & 0.455 & 0.132 & $1.576(1.216-2.042)$ & $0.001^{*}$ \\
Cr (mg/dL) & -0.014 & 0.132 & $0.986(0.761-1.278$ & 0.914 \\
INR & 5.763 & 1.359 & $318.386(22.17-4572.329)$ & $<0.001^{*}$ \\
Platelet (103/uL) & 0.001 & 0.002 & $1.001(0.997-1.006)$ & 0.576 \\
AFP (ng/mL) & 0.000 & 0.000 & $1.000(1.000-1.000)$ & $0.001^{*}$ \\
Cirrhosis (-/+) & 1.131 & 0.422 & $0.10(1.357-7.083)$ & $0.007^{*}$ \\
BCLC staging & 2.394 & 0.411 & $<0.001^{*}$ \\
\hline
\end{tabular}

$(\mathrm{N}=147){ }^{*} p<0.05$ defined as statistically significant.

However, over-expression of IL-6 strongly correlated with Child-Pugh Classification and 6-month mortality in HCCtotal (IL-6: $\mathrm{r}=0.365, \mathrm{P}=0.002$; IL-27: $\mathrm{r}=0.119, \mathrm{P}=0.325$; TNF- $\alpha: r=0.059, P=0.628$; VEGF: $r=0.111, P=0.363$ respectively; IL-6: $\mathrm{r}=0.488, \mathrm{P}<0.001$; IL-27: $\mathrm{r}=-0.109, \mathrm{P}=$ $0.244 ;$ TNF- $\alpha: \mathrm{r}=0.038, \mathrm{P}=0.684$; VEGF: $\mathrm{r}=0.214, \mathrm{P}=$ 0.022 respectively) as well as cirrhotic HCC cases (IL-6: $r=0.376, P=0.002 ;$ IL-27: $r=0.183, P=0.139 ;$ TNF- $\alpha$ : $\mathrm{r}=0.143, \mathrm{P}=0.247$; VEGF: $\mathrm{r}=0.089, \mathrm{P}=0.480$ respectively; IL-6: $\mathrm{r}=0.577, \mathrm{P}<0.001$; IL-27: $\mathrm{r}=-0.036, \mathrm{P}=$ 0.750 ; TNF- $\alpha: \mathrm{r}=0.085, \mathrm{P}=0.451$; VEGF: $\mathrm{r}=0.213, \mathrm{P}=$ 0.059 respectively).

Rather than tissue p-STAT1, tissue p-STAT3 showed predominant immunostain rate and correlated with serum IL-6 expressions among four mediators in HCC To elucidate clinical relationship between tissue p-STAT proteins and serum mediators, we analyzed immunohistochemical expression of p-STAT1 and p-STAT3 in 86 biopsies (52 $\mathrm{CH}$ and $34 \mathrm{HCC}$ cases). Tissue p-STAT3 presented a predominant immunostain rate $(\geqq 10 \%$ immunostaining of hepatocytes) as compared to that of p-STAT1 in all liver $(51 / 86$ versus $25 / 86, \mathrm{P}<0.001)$ and $\mathrm{CH}(32 / 52$ versus $19 / 52, \mathrm{P}=0.003)$ and $\mathrm{HCC}$ specimens (19/34 versus $6 / 34, \mathrm{P}=0.024$ ) (Figures $4 \mathrm{~A}, \mathrm{~B})$. Notably, rising immunoreactivity levels of p-STAT3 instead of pSTAT1 presented positive correlation with over-expression of serum IL-6 rather than IL-27, TNF- $\alpha$, and VEGF not only in overall ( $\mathrm{p}$-STAT3: $\mathrm{r}=0.354, \mathrm{P}=0.001 ; \mathrm{r}=0.116, \mathrm{P}=$ $0.289 ; \mathrm{r}=0.125, \mathrm{P}=0.253 ; \mathrm{r}=0.175, \mathrm{P}=0.109$ respectively) (p-STAT1: $\mathrm{r}=-0.068, \mathrm{P}=0.531 ; \mathrm{r}=-0.085, \mathrm{P}=0.438 ; \mathrm{r}=$ $0.013, \mathrm{P}=0.907 ; \mathrm{r}=-0.057, \mathrm{P}=0.606$ respectively) but also in $\mathrm{HCC}$ cases (p-STAT3: $\mathrm{r}=0.501, \mathrm{P}=0.003 ; \mathrm{r}=0.052, \mathrm{P}=$ $0.770 ; \mathrm{r}=0.019, \mathrm{P}=0.917 ; \mathrm{r}=0.096, \mathrm{P}=0.595$ respectively) (p-STAT1: $\mathrm{r}=0.094, \mathrm{P}=0.597 ; \mathrm{r}=-0.197, \mathrm{P}=0.264$; $\mathrm{r}=-0.093, \quad \mathrm{P}=0.599 ; \quad \mathrm{r}=-0.118, \quad \mathrm{P}=0.511$ respectively) (Figures 4C, D).

\section{Discussion}

Biologic activities of functional mediators activating their downstream special transducer are prominent in regulation of inflammation and carcinogenesis. Still, the evidence of biologic mechanisms and their interrelations with cancer between functional IL-6, IL-27, TNF- $\alpha$, and VEGF with STAT protein is mostly limited to cell line or animal models or non-HCC patients, and poorly understood in liver disease, especially in diverse clinical-pathologic stages of HCC.

The stimulation particularly by hepatitis B or C infection can activate host immune mechanisms to drive serum functional mediators that reflect inflammatory processes and modulate liver regeneration. Indeed, our study only found expressions of serum IL-6, IL-27, and TNF- $\alpha$ rather 

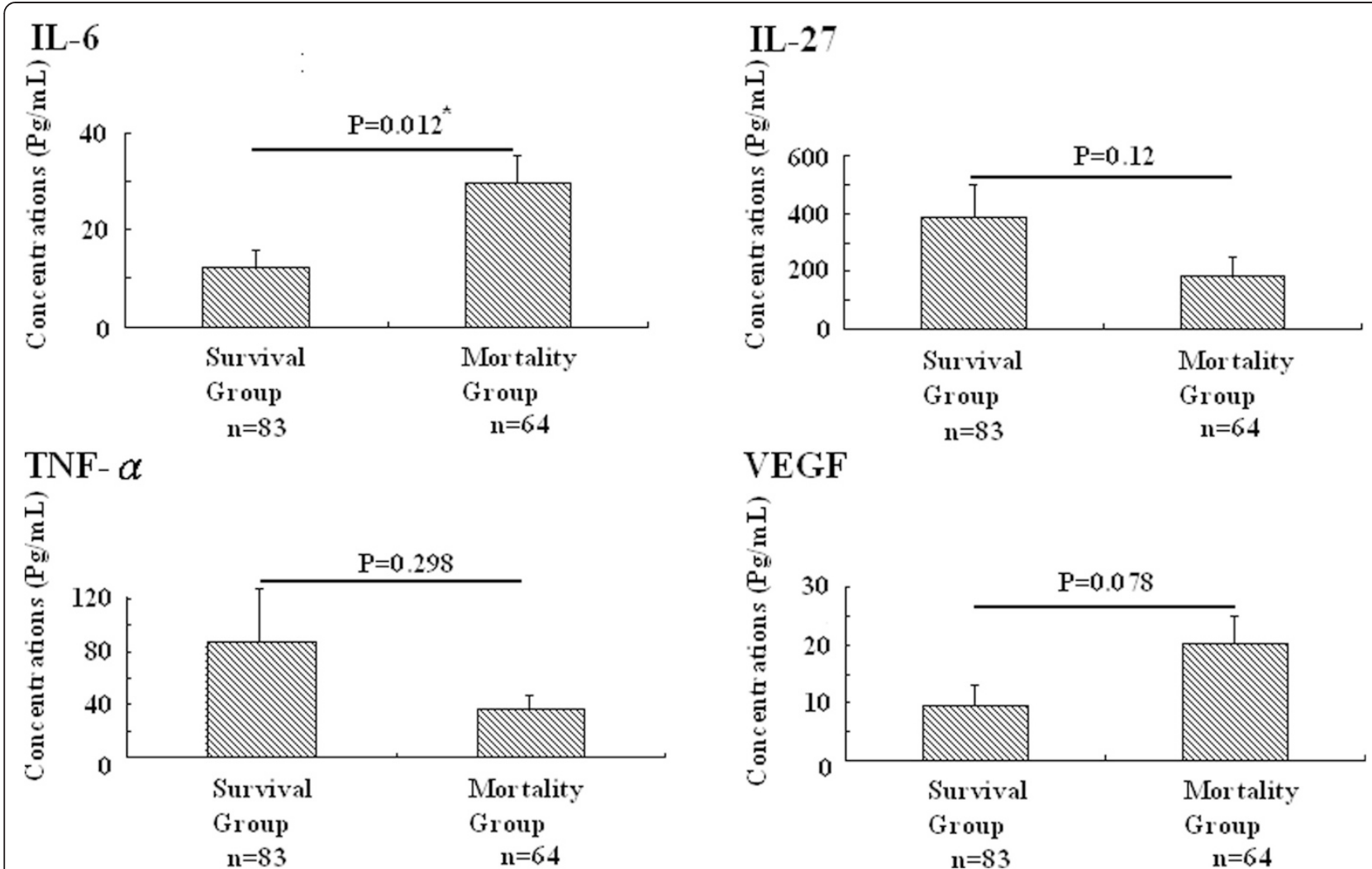

VEGF

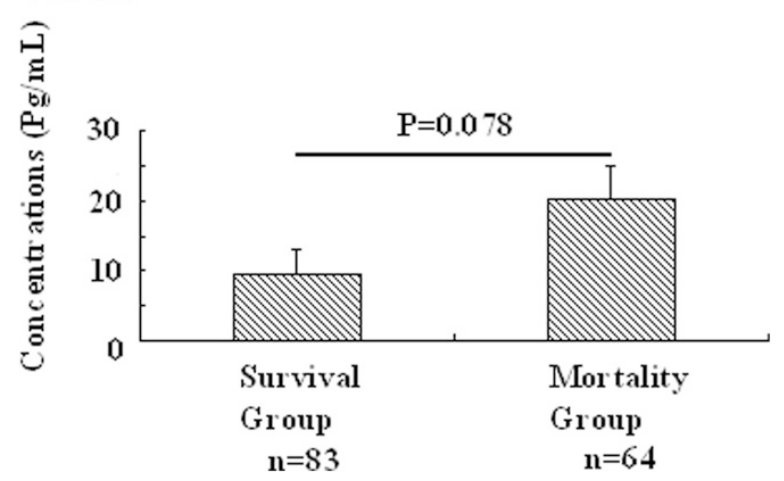

Figure 3 Comparison of four mediators' expression with patient's mortality. Among four mediators, IL-6 presented strongest correlation with 6-month mortality in HCC patients (Figure 3), *P $<0.05$ defined as significant.

than VEGF obvious in $\mathrm{CH}$-total rather than $\mathrm{HG}$ groups (Figure 1), which concurred with previous study: IL-6, IL-27, or TNF- $\alpha$ playing an inflammatory role in regulating hepatocyte proliferation and regeneration, and VEGF playing a major role in pathogenesis of liver cancer $[17,18,20,22,23,26,28]$.

In addition to playing potential inflammation regulators in IL-6, IL-27, and TNF- $\alpha$, our study found these mediators with VEGF expressed strongly in HCC-total or separate $\mathrm{HCC}$ as compared to $\mathrm{CH}$ or $\mathrm{HG}$ cases (Figure 1), which was compatible with prior studies: IL-6, IL-27, and TNF- $\alpha$ could, like VEGF, play a pivotal role in carcinogenesis $[12,16,24,26,28]$. However, correlating their expression with tumor severity (TNM staging system), only IL-6 and VEGF presented a positive trend with tumor progression (Figure 2A). In clinical settings, therapeutic standard and survival prediction of $\mathrm{HCC}$ cases closely relates to levels of the BCLC staging system, combining with tumor severity, liver function, and performance status. We found IL-6 instead of VEGF expressed obvious correlation not only with tumor severity but also with deteriorating liver preservation (Figure 2B). This finding was also supported by parameters associated with impaired liver function: AST $(\mathrm{r}=0.212 ; \mathrm{P}=0.016)$, albumin $(\mathrm{r}=-0.383 ; \mathrm{P}<0.001)$, bilirubin $(\mathrm{r}=0.280 ; \mathrm{P}=0.001)$, INR $(\mathrm{r}=0.299 ; \mathrm{P}<0.001)$, AFP $(\mathrm{r}=0.261 ; \mathrm{P}=0.002)$, and creatinine $(\mathrm{r}=0.181 ; \mathrm{P}=0.032)$ significantly correlated with patient's survival when IL-6 was over-expressed rather than other mediators (Figure 3, Tables 2 and 3). Cirrhosis plays an important role in pathogenesis of liver cancer and patient survival, which also concurred with our result (Table 3). Yet we observed presence or absence of cirrhosis in HCC patients not reflecting expression of IL-6, IL-27, TNF- $\alpha$, and VEGF. This might be complicated and contribute to the liver situation. While correlating among IL-6, IL-27, TNF- $\alpha$, and VEGF with Child-Pugh Classification and 6-month mortality with presence or not of cirrhosis, only IL-6 over-expression strongly correlated with preservation of liver function and 6-month mortality in HCC-total ( $\mathrm{r}=0.365 ; \mathrm{P}=0.002, \mathrm{r}=0.488 ; \mathrm{P}<0.001$ respectively) or cirrhotic HCC $(r=0.376 ; \mathrm{P}=0.002, \mathrm{r}=0.577 ; \mathrm{P}<0.001$ respectively). Findings can explain this discordance. It yields clinical evidence of IL-6 linked with disease progression as compared with IL-27, TNF- $\alpha$, and VEGF in HCC cases.

Several signaling pathways mediating biologic effects of these mediators, and STAT signaling pathway plays an essential component linking cytokine signals to transcription, inducing cell proliferation, protection from apoptosis, tumorigenesis, and higher metastatic potential in diverse 


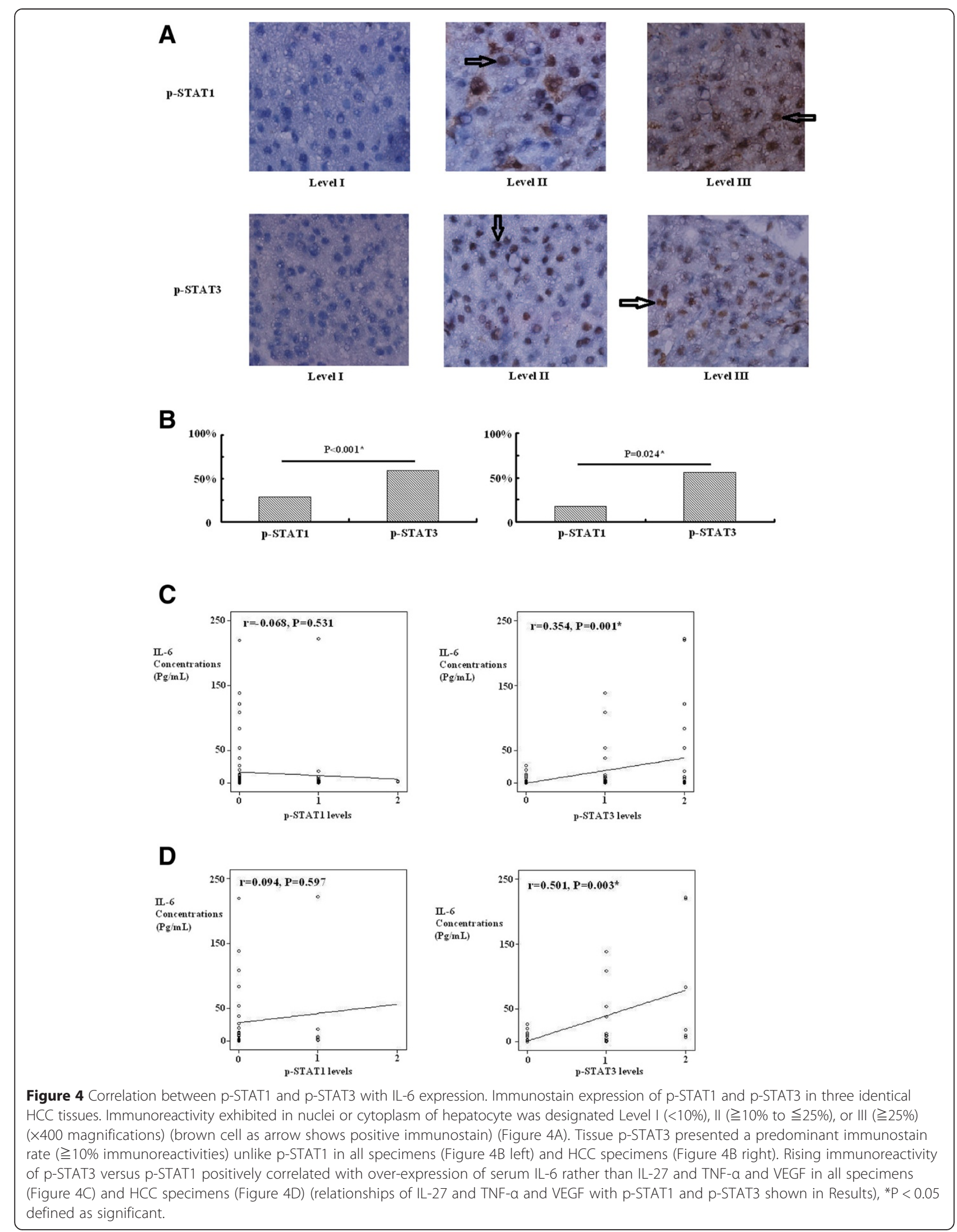


cells [12,16,29-32]. However, clinical correlation between IL-6, IL-27, TNF- $\alpha$, and VEGF with different clinicalpathologic stages of HCC remains limited and warrants further clarification. Both STAT1 and STAT3 proteins have been implicated as essential components linking cytokines signals to transcriptional events in pathogenesis of liver disease [12,16,29]. To correlate STAT1 and STAT3 with their mediators, we examined $\mathrm{CH}$ and $\mathrm{HCC}$ tissues to probe immunostain expressions. Tissues with p-STAT3 expressed a higher immunostain rate overall than $\mathrm{p}$ STAT1 in all liver $(\mathrm{P}<0.001)$, or separate $\mathrm{CH}(\mathrm{P}=0.003)$ and $\mathrm{HCC}$ specimens $(\mathrm{P}=0.024)$ (Figures $4 \mathrm{~A}, \mathrm{~B})$, while rising immunoreactivity level of p-STAT3 reflected significant correlation with IL-6 expression as compared to IL27 , TNF- $\alpha$, and VEGF expression in all patients $(\mathrm{P}=0.001$; $\mathrm{P}=0.289 ; \mathrm{P}=0.253 ; \mathrm{P}=0.109$ respectively $)$ or $\mathrm{HCC}(\mathrm{P}=$ $0.003 ; \mathrm{P}=0.770 ; \mathrm{P}=0.917 ; \mathrm{P}=0.595$ respectively) (Figures $4 \mathrm{C}, \mathrm{D})$. This confirmed a mechanism: IL-6 major through p-STAT3 rather than p-STAT1 pathway affecting severity of inflammation and carcinogenesis in liver disease, particularly in HCC patients $[12,16,29]$. This finding was also concurred with previous study that STAT3 activated by exogenous IL-6 cytokine played a functional role in cholangiocarcinoma development and associated with patient's survival, which belonged to hepatobiliary malignancies [33].

Our study failed to model all participants' liver tissue, this limit based on ethical and safety considerations: HCC patients with decompensated liver disease usually have high hemorrhagic risk. While VEGF presented strong correlation with 6-month mortality (Table 3), it did not reflect as readily as IL-6, which might indicate need for activation via other signal cascades like JAK/ STAT pathway in carcinogenesis [5,26,34]. Fluctuating concentration of serum IL-6, IL-27, TNF- $\alpha$, and VEGF in the host might be argued, but these plasma molecules assessed were not affected by time between blood sampling and centrifuge, according to prior study [35].

\section{Conclusions}

Take together, our findings clearly demonstrate serum IL-6 rather than IL-27, TNF- $\alpha$, and VEGF playing a definite role in liver deterioration and tumor progression, as well as further affecting HCC patient survival. The mechanism of IL-6 biologic activity is chiefly through activation of p-STAT3 instead of p-STAT1 protein in the real world. From functional identification of IL-6/pSTAT3 pathways, we believe ELISA detection of circulating IL-6 and immunostain of tissue p-STAT3 as biomarker combined with current clinical biochemical data or images can provide clinicians with useful references for prognosis. Such an attractive immunotherapeutic strategy would reduce or prevent mortality in the future.

\section{Competing interests}

The authors declare that they have no competing interests.

\section{Authors' contributions}

JTK carried out the molecular studies, cases collection, and wrote the manuscript. CLF and CJY participated in the design of the study, and cases collection. SMT participated in the immunoassays and performed the statistical analysis. PNH conceived of the study and participated in its design. YLC conceived of the study and helped to draft the manuscript. YYW conceived of the study, and participated in its design and coordination and helped to finish the manuscript. All authors read and approved the final manuscript.

\section{Acknowledgements}

This study was supported by grants from the National Science Council, Taiwan, and China Medical University, Taiwan (CMU100-NTU-05). We are sincerely grateful to all participants. We also thank all research assistants of the Liver Unit for excellent technical assistance in data collection, case enrollment, and serologic assay.

\section{Author details}

${ }^{1}$ School of Medicine, China Medical University, Taichung, Taiwan. ${ }^{2}$ Graduate Institute of Clinical Medical Science, China Medical University, Taichung, Taiwan. ${ }^{3}$ Department of Internal Medicine, Division of Hepato-Gastroenterology, China Medical University Hospital, Taichung, Taiwan. ${ }^{4}$ Graduate Institute of Clinical Medical Science, Chang Gung University, Tao-Yuan, Taiwan. ${ }^{5}$ Graduate Institute of Immunology, College of Medicine, National Taiwan University, Taipei, Taiwan. ${ }^{6}$ School of Medicine, Kaohsiung Medical University, Kaohsiung, Taiwan. ${ }^{7}$ Department of General Surgery, Changhua Christian Hospital, Changhua, Taiwan. ${ }^{8}$ Department of Medical Laboratory Science and Biotechnology, China Medical University, No. 91, Hsueh-Shih Rd., Taichung 404, Taiwan.

Received: 3 March 2015 Accepted: 8 April 2015

Published online: 25 April 2015

\section{References}

1. Garcia M, Jemal A, Ward EM, Center MM, Hao Y, Siegel RL, Thun MJ. Global Cancer Facts and Figures 2007 [http://www.cancer.org]

2. Lee WM. Hepatitis B virus infection. N Engl J Med. 1997;337:1733-45.

3. Lauer GM, Walker BD. Hepatitis C virus infection. N Engl J Med. 2001;345:41-52.

4. Bosch FX, Ribes J, Díaz M, Cléries R. Primary liver cancer: worldwide incidence and trends. Gastroenterology. 2004;127:S5-16.

5. Singal A, Volk ML, Waljee A, Salgia R, Higgins P, Rogers MA, et al. Meta-analysis: surveillance with ultrasound for early-stage hepatocellular carcinoma in patients with cirrhosis. Aliment Pharmacol Ther. 2009;30:37-47.

6. Llovet JM. Updated treatment approach to hepatocellular carcinoma. J Gastroenterol. 2005;40:225-35.

7. Semela D, Dufour JF. Angiogenesis and hepatocellular carcinoma. J Hepatol. 2004:41:864-80

8. Villanueva A, Hoshida Y, Toffanin S, Lachenmayer A, Alsinet C, Savic R, et al. New strategies in hepatocellular carcinoma: genomic prognostic markers. Clin Cancer Res. 2010;16:4688-94.

9. Llovet JM, Pena CE, Lathia CD, Shan M, Meinhardt G, Bruix J, et al. Plasma biomarkers as predictors of outcome in patients with advanced hepatocellular carcinoma. Clin Cancer Res. 2012;18:2290-300.

10. Llovet JM, Ricci S, Mazzaferro V, Hilgard P, Gane E, Blanc JF, et al. Sorafenib in advanced hepatocellular carcinoma. N Engl J Med. 2008;359:378-90.

11. Cheng AL, Kang YK, Chen Z, Tsao CJ, Qin S, Kim JS, et al. Efficacy and safety of sorafenib in patients in the Asia-Pacific region with advanced hepatocellular carcinoma: a phase III randomised, double-blind, placebo-controlled trial. Lancet Oncol. 2009;10:25-34.

12. Johnson C, Han Y, Hughart N, McCarra J, Alpini G, Meng F. Interleukin-6 and its receptor, key players in hepatobiliary inflammation and cancer. Transl Gastrointest Cancer. 2012;1:58-70.

13. Trinchieri G, Pflanz S, Kastelein RA. The IL-12 family of heterodimeric cytokines: new players in the regulation of T cell responses. Immunity. 2003;19:641-4.

14. Das $L$, Vinayak M. Long term effect of curcumin down regulates expression of TNF- $a$ and IL-6 via modulation of ETS and NF-KB transcription factor in liver of lymphoma bearing mice. Leuk Lymphoma 2014 Feb 4. [Epub ahead of print] 
15. Ferrara N, Gerber HP, LeCouter J. The biology of VEGF and its receptors. Nat Med. 2003:9:669-76.

16. Guo Y, Xu F, Lu TJ, Duan ZF, Zhang Z. Interleukin-6 signaling pathway in targeted therapy for cancer. Cancer Treat Rev. 2012;38:904-10.

17. Cressman DE, Greenbaum LE, DeAngelis RA, Ciliberto G, Furth EE, Poli V, et al. Liver failure and defective hepatocyte regeneration in interleukin-6deficient mice. Science. 1996;274:1379-83.

18. Kao JT, Lai HC, Tsai SM, Lin PC, Chuang PH, Yu CJ, et al. Rather than interleukin-27, interleukin-6 expresses positive correlation with liver severity in naïve hepatitis B infection patients. Liver Int. 2012;32:928-36.

19. Li K, Li NL, Wei D, Pfeffer SR, Fan M, Pfeffer LM. Activation of chemokine and inflammatory cytokine response in hepatitis $C$ virus-infected hepatocytes depends on toll-like receptor 3 sensing of hepatitis $C$ virus double-stranded RNA intermediates. Hepatology. 2012;55:666-75.

20. Bender $\mathrm{H}$, Wiesinger MY, Nordhoff C, Schoenherr C, Haan C, Ludwig S, et al. Interleukin-27 displays interferon-gamma-like functions in human hepatoma cells and hepatocytes. Hepatology. 2009;50:585-91.

21. Shimizu M, Shimamura M, Owaki T, Asakawa M, Fujita K, Kudo M, et al. Antiangiogenic and antitumor activities of IL-27. J Immunol. 2006;176:7317-24.

22. Moncada S, Higgs A. The L-arginine-nitric oxide pathway. N Engl J Med. 1993;329:2002-12

23. Mookerjee RP, Sen S, Davies NA, Hodges SJ, Williams R, Jalan R. Tumour necrosis factor is an important mediator of portal and systemic haemodynamic derangements in alcoholic hepatitis. Gut. 2003;52:1182-7.

24. Jiao SF, Sun K, Chen XJ, Zhao X, Cai N, Liu YJ, et al. Inhibition of tumor necrosis factor alpha reduces the outgrowth of hepatic micrometastasis of colorectal tumors in mouse model of liver ischemia-reperfusion injury. J Biomed Sci. 2014:21:1.

25. Leung DW, Cachiane G, Kuang WJ, Goeddel DV, Ferrara N. Vascular endothelial growth factor is a secreted angiogenic mitogen. Science. 1989;246:1306-9.

26. Suzuki K, Hayashi N, Miyamoto Y, Yamamoto M, Ohkawa K, Ito Y, et al. Expression of vascular permeability factor/vascular endothelial growth factor in human hepatocellular carcinoma. Cancer Res. 1996;56:3004-9.

27. Wilhelm SM, Carter C, Tang L, Wilkie D, McNabola A, Rong H, et al. BAY 43-9006 exhibits broad spectrum oral antitumor activity and targets the Raf/Mek/Erk pathway and receptor tyrosine kinases involved in tumor progression and angiogenesis. Cancer Res. 2004;64:7099-109.

28. Ribatti D, Vacca A, Nico B, Sansonno D, Dammacco F. Angiogenesis and anti-angiogenesis in hepatocellular carcinoma. Cancer Treat Rev. 2006;32:437-44

29. Gao B, Wang H, Lafdil F, Feng D. STAT proteins-Key regulators of anti-viral responses, inflammation, and tumorigenesis in the liver. J Hepatol. 2012:57:430-41.

30. Slattery ML, Lundgreen A, Kadlubar SA, Bondurant KL, Wolff RK. JAK/STAT/ SOCS-signaling pathway and colon and rectal cancer. Mol Cancinog. 2013;52:155-66.

31. Chen Y, Lan Q, Zheng T, Zhao N, Holford TR, Lerro C, et al. Polymorphisms in JAK/STAT signaling pathway genes and risk of non-Hodgkin lymphoma. Leuk Res. 2013:37:1120-4.

32. Fazilaty $H$, Mehdipour P. Genetics of breast cancer bone metastasis: a sequential multistep pattern. Clin Exp Metastasis 2014 Feb 4. [Epub ahead of print]

33. Dokduang $H$, Techasen A, Namwat N, Khuntikeo N, Pairojkul C, Murakami $Y$, et al. STATs profiling reveals predominantly-activated STAT3 in cholangiocarcinoma genesis and progression. J Hepatobiliary Pancreat Sci. 2014;21:767-76.

34. Wei LH, Kuo ML, Chen CA, Chou CH, Lai KB, Lee CN, et al. Interleukin-6 promotes cervical tumor growth by VEGF dependent angiogenesis via a STAT3 pathway. Oncogene. 2003:22:1517-27.

35. Webb NJ, Bottomley MJ, Watson CJ, Brenchley PE. Vascular endothelial growth factor (VEGF) is released from platelets during blood clotting: implications for measurement of circulating VEGF levels in clinical disease. Clin Sci (Lond). 1998;94:395-404.

\section{Submit your next manuscript to BioMed Central and take full advantage of:}

- Convenient online submission

- Thorough peer review

- No space constraints or color figure charges

- Immediate publication on acceptance

- Inclusion in PubMed, CAS, Scopus and Google Scholar

- Research which is freely available for redistribution

Submit your manuscript at www.biomedcentral.com/submit 